\title{
Exact Ground State Wave Functions for N Anyons in a Magnetic Field on the Torus
}

\author{
Ansar Fayyazuddin \\ Institute of Theoretical Physics \\ Stockholm University \\ Vanadisvägen 9 \\ S-113 46 Stockholm, Sweden
}

March 1993

\begin{abstract}
The complete set of ground state wave functions for $\mathrm{N}$ anyons in an external magnetic field on the torus is found. The cases when the filling factor is less than or equal to one are considered. The single valued description of anyons is employed through out by coupling bosons to a Chern-Simons field. At the end, the Chern-Simons interaction is removed by a singular gauge transformation as a result of which the wave functions become multi-component in agreement with other studies.
\end{abstract}

USITP-93-07 
Anyons [1], particles obeying fractional statistics, have been the subject of intense study in the past few years. Besides the purely theoretical interest in fractional statistics a new motivation has been provided by the Laughlin theory of the fractional quantum hall effect (FQHE) 2]. It was realized some time ago that certain excitations of the Laughlin state are anyonic [3]. Anyons are, therefore, characteristic of the elusive Laughlin state. An understanding of anyons in a magnetic field may help to provide, in turn, an understanding of the rich structure of the Laughlin state. This has been the motivation behind much of the study of anyons in magnetic fields. Some exact solutions on the plane can be found in ref [ [].

In this letter we study the problem of anyons in an external magnetic field when the global topology is that of a torus. These, somewhat special, conditions put rather strict constraints on the wave functions. We will consider anyons on a torus which we parametrize by a rectangle of dimensions $L_{1} \times L_{2}$ in the $\mathrm{x}-\mathrm{y}$ plane with opposite sides identified. It is now known that anyons on the torus can be equivalently studied by coupling bosons to a Chern-Simons field. The anyonic solutions can be recovered from the bosonic theory by a singular gauge transformation. The single valued description of anyons will be employed till the end where we recover the multi-valued, multi-component wave functions by performing a singular gauge transformation.

The following notation will be used through out this letter: $x_{\gamma}, y_{\gamma}$ denote the coordinates of particle $\gamma$, and $X=\sum x_{\gamma}, Y=\sum y_{\gamma}$ the center of mass coordinates for the entire system. The complex coordinates $z_{\gamma} \equiv\left(x_{\gamma}+i y_{\gamma}\right) / L_{1}$ will sometimes be collected in a vector $\vec{z} \equiv z_{\gamma} \hat{e}_{\gamma}$, where the $\hat{e}_{\gamma}$ denotes a vector with $N_{A}$ entries all of which are zero except the $\gamma$ th which is a one. Use will also be made of the vector $\hat{e} \equiv \sum_{\alpha=1}^{N_{A}} \hat{e}_{\alpha}$. The Chern-Simons coupling constant $\kappa=p / q>0$, where $p, q$ are relatively prime integers, is related to the statistical phase $\theta$ in the anyon description by $\theta=\pi / \kappa$. The total flux $N_{\Phi}=\omega-N_{A} / \kappa$, where $\omega=B L_{1} L_{2} / 2 \pi$ and $N_{A}$ is the number of particles, is an integer consistent with the Dirac quantization condition. Notice that we need not require the Chern-Simons and magnetic flux to be integer seperately since only the holonomy associated with the sum of the gauge fields is an observable. Finally, $N$ denotes the greatest common denominator of $N_{\Phi}$ and $N_{A}, s \equiv N_{A} / N, r \equiv N_{\Phi} / N$; needless to say, these definitions are only sensible when $N_{\Phi} \neq 0$ which should be assumed unless explicitly stated otherwise. $a, b$ are defined by $s=a p+b$ with $0 \leq a, 0 \leq b<p$. The modular parameter $\tau$ appearing in the theta functions is defined to be $\tau=i L_{2} / L_{1}$.

The following solution to the Chern-Simons constraint will be used [11] (other solutions are discussed in [12, 6, 5]):

$$
\begin{aligned}
& a_{\alpha x}=\frac{\eta}{L_{1}}+\frac{2 \pi}{\kappa L_{1} L_{2}} Y-\frac{1}{\kappa} \sum_{\mu \neq \alpha} \operatorname{Im} \frac{\theta_{1}^{\prime}\left(z_{\alpha}-z_{\mu} \mid \tau\right)}{\theta_{1}\left(z_{\alpha}-z_{\mu} \mid \tau\right)} \\
& a_{\alpha y}=\frac{\zeta}{L_{2}}-\frac{1}{\kappa} \sum_{\mu \neq \alpha} \operatorname{Re} \frac{\theta_{1}^{\prime}\left(z_{\alpha}-z_{\mu} \mid \tau\right)}{\theta_{1}\left(z_{\alpha}-z_{\mu} \mid \tau\right)}
\end{aligned}
$$

$\eta$ and $\zeta$ are the part of the Chern-Simons gauge field not determined by the gauss law constraint generated by the Chern-Simons term; they satisfy the commutation relation:

$$
[\eta, \zeta]=i \frac{2 \pi}{\kappa}
$$

Thus $\zeta$ can be represented as $\zeta=-i \frac{2 \pi}{\kappa} \partial / \partial_{\eta}$. For the external gauge field $\vec{A}$ the following gauge will be fixed:

$$
\vec{A}_{\alpha}=-B y_{\alpha} \hat{i}
$$


The Hamiltonian for this system is:

$$
\begin{aligned}
H & =-\frac{1}{2 m} \sum_{\alpha=1}^{N_{A}} \vec{D}_{\alpha} \cdot \vec{D}_{\alpha} \\
D_{\alpha x} & =\frac{\partial}{\partial x_{\alpha}}-i\left[\frac{\eta}{L_{1}}+\frac{2 \pi}{\kappa L_{2} L_{1}} Y-B y_{\alpha}-\frac{1}{\kappa} \sum_{\mu \neq \alpha} \operatorname{Im} \frac{\theta_{1}^{\prime}\left(z_{\alpha}-z_{\mu} \mid \tau\right)}{\theta_{1}\left(z_{\alpha}-z_{\mu} \mid \tau\right)}\right] \\
D_{\alpha y} & =\frac{\partial}{\partial y_{\alpha}}-\frac{2 \pi}{\kappa L_{2}} \frac{\partial}{\partial \eta}+\frac{i}{\kappa} \sum_{\mu \neq \alpha} \operatorname{Re} \frac{\theta_{1}^{\prime}\left(z_{\alpha}-z_{\mu} \mid \tau\right)}{\theta_{1}\left(z_{\alpha}-z_{\mu} \mid \tau\right)}
\end{aligned}
$$

Since neither the Chern-Simons gauge field nor the external gauge field are globally defined on the torus the hamiltonian is not periodic under both cycles of the torus. It is easily checked that the gauge fields are periodic under $x_{\alpha} \rightarrow x_{\alpha}+L_{1}$ but not under $y_{\alpha} \rightarrow y_{\alpha}+L_{2}$. Under the latter the hamiltonian changes by a gauge transformation which is well defined on the torus if the total flux $N_{\Phi}$ is an integer. Whenever $N_{\Phi} \neq 0$, gauge invariant observables are well defined only if the wave function changes as follows 円:

$$
\begin{aligned}
& \psi\left(x_{\alpha}+L_{1}\right)=\psi\left(x_{\alpha}\right) \\
& \psi\left(y_{\alpha}+L_{2}\right)=e^{-i \frac{2 \pi x_{\alpha} N_{\Phi}}{L_{1}}} \psi\left(y_{\alpha}\right)
\end{aligned}
$$

upto a possible global $U(1)$ rotation. In addition to translation invariance of observables, one has to impose invariance under large gauge transformations $\|6\|$ : i.e. covariance of the wave functions under $U_{1}=\exp 2 \pi \partial_{\eta} \exp -i 2 \pi X / L_{1}$ and $U_{2}=\exp 2 \pi \partial_{\zeta} \exp -i 2 \pi Y / L_{2}=\exp -i \kappa\left(\eta+2 \pi Y / \kappa L_{2}\right)$. The $U_{i}$ satisfy the following commutation relation:

$$
U_{1} U_{2}=U_{2} U_{1} e^{i 2 \pi \kappa}
$$

From now on we will work in a basis in which $U_{2}$ is diagonal, the commutation relation then determines the action of $U_{1}$ upto a global phase:

$$
\begin{aligned}
U_{2} \psi_{l} & =e^{-i \beta-i 2 \pi \kappa l} \psi_{l} \\
U_{1} \psi_{l} & =e^{i \alpha} \psi_{l-1}
\end{aligned}
$$

It should be emphasized that different components of the $q$ dimensional representation of the group of large gauge transformations represent the same physical state. They are gauge copies of one another.

Having stated the quasi-periodicity conditions for the wave functions in both space and the topological part of the gauge field, we now seek eigenstates of the Hamiltonian which satisfy these conditions. First we expand the wave function in the coordinates: $x_{\gamma}, y_{\gamma}$ and $v \equiv \eta+2 \pi Y / \kappa L_{2}$. Notice that the dependence on $\eta$ is through $v$ only. Write $\psi_{l}$ as:

$$
\psi_{l}=e^{-\frac{\pi \omega}{L_{1} L 2} \sum_{\gamma} y_{\gamma}^{2}} e^{v Y / L_{1}} \prod_{\gamma<\delta}\left|\theta_{1}\left(z_{\gamma}-z_{\delta} \mid \tau\right)\right|^{\frac{1}{\kappa}} \phi_{l}(x, y, v)
$$

Covariance under large gauge transformations and $[v, H]=0$ allow for the following general expression for $\phi_{l}$ :

$$
\phi_{l}(x, y, v)=\sum_{n=0}^{p-1} \sum_{j=-\infty}^{\infty} e^{i \vec{z} \cdot \hat{e}(l+j q)} e^{i \alpha(l+j q+n / \kappa)} F_{n}(x, y) \delta(\kappa v-2 \pi \kappa l-\beta-2 \pi(j p+n))
$$

\footnotetext{
${ }^{1}$ Only the coordinate indicated is transformed the others are held fixed.
} 
Where the $F_{n}$ are bosonic functions independent of $v$. Note that for any holomorphic $F_{n}$ the $\psi_{l}$ are eigenstates of the Hamiltonian with the lowest possible energy eigenvalue.

We define the magnetic translation operators:

$$
\begin{aligned}
t_{\alpha}\left(m \vec{L}_{1}\right) & =e^{m L_{1} \frac{\partial}{\partial x} \alpha} \\
t_{\alpha}\left(n \vec{L}_{2}\right) & =e^{i 2 \pi n N_{\Phi} x_{\alpha} / L_{1}} e^{n L_{2} \frac{\partial}{\partial y}} \alpha
\end{aligned}
$$

These operators commute among themselves and with the Hamiltonian only when $m, n$ are integers. When $N_{\Phi}$ vanishes the translation operators commute among themselves for arbitrary argument but not with the hamiltonian except in the singular limit in which $p \rightarrow \infty$. Quasi-periodicity under lattice translations is conveniently expressed as invariance of the wave functions under these translation operators for $m=n=1$. The center of mass translation operators

$$
T\left(\vec{L}_{i}\right) \equiv \prod_{\alpha} t_{\alpha}\left(\vec{L}_{i} / N\right)
$$

commute with the $t_{\alpha}\left(\vec{L}_{i}\right)$, the hamiltonian and among themselves. This gives a maximal set of translation operators that can be simultaneously diagonalized [9]. Since we are dealing here with a system of identical particles $t_{\alpha}=t_{\beta}$ on the Hilbert space of wave functions. Explicitly, magnetic translation invariance

$$
t_{\alpha}\left(\vec{L}_{i}\right) \psi_{l}(\{x, y\}, v)=\psi_{l}(\{x, y\}, v)
$$

imposes the following conditions on the holomorphic $F_{n}(\vec{z})$ :

$$
\begin{gathered}
F_{n}\left(\vec{z}+\hat{e}_{\gamma}\right)=F_{n}(\vec{z}) \\
e^{i 2 \pi N_{\Phi} \hat{e}_{\gamma} \cdot \vec{z}+i \pi \tau\left(N_{\Phi}+\frac{1}{\kappa}\right)-i \frac{\tau}{\kappa}(2 \pi n+\beta)+i \frac{\alpha}{\kappa}} F_{n}\left(\vec{z}+\tau \hat{e}_{\gamma}\right)=F_{n-1}(\vec{z})(\text { for } n>0) \\
e^{i 2 \pi\left(N_{\Phi} \hat{e}_{\gamma}+q \hat{e}\right) \cdot \vec{z}+i \pi \tau\left(N_{\Phi}+\frac{1}{\kappa}\right)-i \frac{\tau}{\kappa} \beta+i \frac{\alpha}{\kappa}} F_{0}\left(\vec{z}+\tau \hat{e}_{\gamma}\right)=F_{p-1}(\vec{z})
\end{gathered}
$$

By repeated application of $t_{\alpha}\left(\vec{L}_{2}\right)$ the following relation can be derived:

$$
e^{i 2 \pi\left(p N_{\Phi} \hat{e}_{\gamma}+q \hat{e}\right) \cdot \vec{z}+i \pi \tau p^{2}\left(N_{\Phi}+\frac{1}{\kappa}\right)-i p \frac{\tau}{\kappa}(2 \pi n+\beta)+i q \alpha} F_{n}\left(\vec{z}+p \tau \hat{e}_{\gamma}\right)=F_{n}(\vec{z})
$$

The next step in constructing a complete set of functions $F_{n}$ is best motivated by pausing here to state some definitions and a theorem [7] of which use will be made later.

Definition: Given $\Omega$, a $g \times g$ complex, symmetric matrix such that $\operatorname{Im} \Omega$ is positive definite (in the mathematical literature the space of such matrices $\mathcal{M}_{g}$ is called the Siegel upper-half-space), we define the theta function $\theta\left[\begin{array}{l}\vec{a} \\ \vec{b}\end{array}\right](\vec{z}, \Omega)$ with characteristics $(\vec{a}, \vec{b})$ as:

$$
\theta\left[\begin{array}{l}
\vec{a} \\
\vec{b}
\end{array}\right](\vec{z}, \Omega)=\sum_{\vec{n} \in Z^{g}} \exp [i \pi(\vec{n}+\vec{a}) \cdot \Omega(\vec{n}+\vec{a})+i 2 \pi(\vec{n}+\vec{a}) \cdot(\vec{z}+\vec{b})]
$$

The positive definiteness of $\operatorname{Im} \Omega$ guarantees uniform and absolute convergence of the series defining the theta function. These functions satisfy the following quasi-periodicity conditions:

$$
\theta\left[\begin{array}{c}
\vec{a} \\
\vec{b}
\end{array}\right](\vec{z}+\vec{m}, \Omega)=e^{i 2 \pi \vec{a} \cdot \vec{m}} \theta\left[\begin{array}{l}
\vec{a} \\
\vec{b}
\end{array}\right](\vec{z}, \Omega)
$$




$$
\begin{gathered}
\theta\left[\begin{array}{c}
\vec{a} \\
\vec{b}
\end{array}\right](\vec{z}+\Omega \vec{m}, \Omega)=e^{-i 2 \pi \vec{b} \cdot \vec{m}} \exp (-i \pi \vec{m} \cdot \Omega \vec{m}-i 2 \pi \vec{m} \cdot \vec{z}) \theta\left[\begin{array}{c}
\vec{a} \\
\vec{b}
\end{array}\right](\vec{z}, \Omega) \\
\theta\left[\begin{array}{c}
\vec{a}+\vec{m} \\
\vec{b}+\vec{n}
\end{array}\right](\vec{z}, \Omega)=e^{i 2 \pi \vec{a} \cdot \vec{n}} \theta\left[\begin{array}{c}
\vec{a} \\
\vec{b}
\end{array}\right](\vec{z}, \Omega)
\end{gathered}
$$

whenever $\vec{m}, \vec{n} \in Z^{g}$.

Definition: Fix $\Omega \in \mathcal{M}_{g}$. Then an entire function $f(\vec{z})$ on $C^{g}$ is $L_{\Omega}$-quasi-periodic of weight $\ell$ if

$$
\begin{aligned}
f(\vec{z}+\vec{m}) & =f(\vec{z}) \\
f(\vec{z}+\Omega \vec{m}) & =\exp (-i \pi \ell \vec{m} \cdot \Omega \vec{m}-i 2 \pi \ell \vec{m} \cdot \vec{z}) f(\vec{z})
\end{aligned}
$$

for all $\vec{m} \in Z^{g}$. Let $R_{\ell}^{\Omega}$ be the vector space of such functions $f$.

Theorem: Fix $\Omega \in \mathcal{M}_{g}$. Then a basis of $R_{\ell}^{\Omega}$ is given by:

$$
f_{\vec{c}}=\theta\left[\begin{array}{c}
\overrightarrow{0} \\
\vec{c} \\
\hline \ell
\end{array}\right]\left(\vec{z}, \ell^{-1} \Omega\right)
$$

for $c_{i} \in Z, 0 \leq c_{i}<\ell$.

Though this theorem is very powerful its proof is simple [7]: it immediately follows by noting that the first condition allows one to expand the function in a Fourier series and that the second condition gives a recursion relation between the coefficients of the series. The most general solution for the coefficients establishes the theorem. Of course a different representation for the basis elements is always possible.

Returning to the problem at hand, we would like to find a basis of functions $F_{n}$ by using the above theorem. To this end we rewrite the quasi-periodicity relation for the $F_{n}$ as:

$$
\begin{aligned}
& F_{n}\left(\vec{z}+p \tau \hat{e}_{\gamma}\right)=F_{n}\left(\vec{z}+\Omega^{\prime} \vec{m}_{\gamma}\right) \\
= & \exp \left(-i \pi \ell \vec{m}_{\gamma} \cdot \Omega^{\prime} \vec{m}_{\gamma}-i 2 \pi \ell \vec{m}_{\gamma} \cdot\left(\vec{z}-\frac{\tau}{\omega \kappa}\left(n+\frac{\beta}{2 \pi}\right) \hat{e}+\frac{\alpha}{2 \pi \omega \kappa}\right)\right) F_{n}(\vec{z})
\end{aligned}
$$

Comparing with equation (18) gives:

$$
\ell \vec{m}_{\gamma}=p N_{\Phi} \hat{e}_{\gamma}+q \hat{e}
$$

and

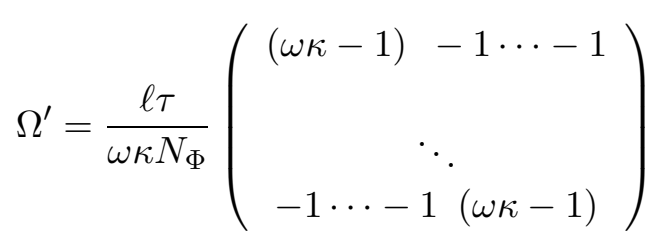

$\operatorname{Im} \Omega^{\prime}$ is positive definite only if $\omega \kappa-N_{A}>0$ which we assume from now on. This is essentially the condition that the anyonic generalization of the Landau level filling factor, $N_{A} / \omega \kappa$, be less than one (the case where $\omega \kappa=N_{A}$ will be dealt with seperately below). To fix $\Omega^{\prime}$, require that for any $\vec{n} \in Z^{N_{A}}$

$$
\tau^{-1} \Omega^{\prime} \vec{n} \in p Z^{N_{A}}
$$

The choice $\ell=p^{2} \omega N_{\Phi}$ fullfills the above condition and $F_{n}$ belongs to $R_{\ell}^{\Omega^{\prime}}$. The theorem then provides us with a basis of $R_{\ell}^{\Omega^{\prime}}$. Using these functions we can construct the $F_{n}$ which satisfy 
rather more stringent conditions than a general member of $R_{\ell}^{\Omega^{\prime}}$. A basis for $R_{\ell}^{\Omega^{\prime}}$ is given by the set of functions:

$$
g_{n, \vec{c}}=\theta\left[\begin{array}{c}
\overrightarrow{0} \\
\vec{c} \\
\ell
\end{array}\right]\left(\vec{z}-\frac{\tau}{\omega \kappa}\left(n+\frac{\beta}{2 \pi}\right) \hat{e}+\frac{\alpha}{2 \pi \omega \kappa} \hat{e}, \Omega\right)
$$

where $\Omega=1 / \ell \Omega^{\prime}$.

We now express the functions $F_{n}$ in the above basis:

$$
F_{n}(\vec{z})=\sum_{\vec{c}} D_{\vec{c}, n} \theta\left[\begin{array}{c}
\overrightarrow{0} \\
\frac{\vec{c}}{\ell}
\end{array}\right]\left(\vec{z}-\frac{\tau}{\omega \kappa}\left(n+\frac{\beta}{2 \pi}\right) \hat{e}+\frac{\alpha}{2 \pi \omega \kappa} \hat{e}, \Omega\right)
$$

Imposing the quasi-periodicity conditions on the $F_{n}$ relate the coefficients:

$$
D_{\vec{c}, p-1}=D_{\vec{c}, 0} e^{-i 2 \pi\left(q|c|+N_{\Phi} c_{\gamma}\right) / \ell} e^{\frac{i \pi \tau}{\omega \kappa^{2}}(p-1)^{2}} e^{i \tau \beta N_{A}(p-1) / \omega \kappa^{2}} e^{-i \alpha N_{A}(p-1) / \omega \kappa^{2}}
$$

with the requirement:

$$
e^{-i 2 \pi\left(q|c|+p N_{\Phi} c_{\gamma}\right) / \ell}=1
$$

(where the notation $|c|=\sum_{\gamma=1}^{N_{A}} c_{\gamma}$ has been introduced) and

$$
D_{\vec{c}, n-1}=D_{\vec{c}, n} e^{-i 2 \pi N_{\Phi} c_{\gamma} / \ell} e^{\frac{i \pi \tau}{\omega \kappa^{2}}} e^{\frac{-i 2 \pi \tau N_{A}}{\omega \kappa^{2}}(n+\beta / 2 \pi)} e^{i \alpha N_{A} / \omega \kappa^{2}}
$$

Further, diagonalizing the center of mass translation operators $T\left(\vec{L}_{2}\right)$ with eigenvalue $\exp i 2 \pi k_{2} / N$ gives for $n<b$ :

$$
D_{\vec{c}, n+p-b}=D_{\vec{c}, n} e^{-i 2 \pi k_{2} / N} e^{-i 2 \pi(p w+q N(p-b))|c| / \ell p N} e^{\frac{i \pi \tau}{\omega \kappa^{2}}(p-b)^{2}} e^{\frac{i 2 \pi \tau N_{A}}{\omega \kappa^{2}}(n+\beta / 2 \pi)(p-b)} e^{\frac{-i \alpha N_{A}}{\omega \kappa^{2}}(p-b)}
$$

and for $n>b$ :

$$
D_{\vec{c}, n-b}=D_{\vec{c}, n} e^{-i 2 \pi k_{2} / N} e^{-i 2 \pi(p w-q N b)|c| / \ell p N} e^{\frac{i \pi \tau}{\omega \kappa^{2}} b^{2}} e^{\frac{-i 2 \pi \tau N_{A}}{\omega \kappa^{2}}(n+\beta / 2 \pi) b} e^{\frac{i \alpha N_{A}}{\omega \kappa^{2}} b}
$$

Diagonalizing $T\left(\vec{L}_{1}\right)$ with eigenvalue $\exp i 2 \pi k_{1} / N$ gives:

$$
D_{\vec{c}, n}=D_{\vec{c}+p^{2} r \omega \hat{e}, n} e^{i 2 \pi k_{1} / N}
$$

Finally, requiring that the action of the translation operators be independent of particle index i.e. $t_{\alpha}=t_{\beta}$ (identical particles) imposes the constraint:

$$
\exp -i 2 \pi N_{\Phi} c_{\gamma} / \ell=\exp -i 2 \pi N_{\Phi} c_{\delta} / \ell
$$

for any $\gamma, \delta$.

Using these relations one can express the eigenvalue $\exp i 2 \pi k_{2} / N$ in terms of $\vec{c}$ :

$$
e^{i 2 \pi k_{2} / N}=e^{-i 2 \pi s N_{\Phi} c_{\gamma} / \ell} e^{i 2 \pi|c| r / \ell}
$$

Notice that due to the constraints on $\vec{c}$, the right hand side is an Nth root of unity and is independent of the index $\gamma$. Given $\vec{c}$ satisfying the above constraints the coefficients can be solved for giving the most general form:

$$
D_{\vec{c}, n}=d_{\vec{c}} e^{\frac{i \pi}{\kappa^{2}}\left(n+\frac{\beta}{2 \pi}\right)^{2} \hat{e} \cdot \Omega \hat{e}} e^{-i 2 \pi(n / \kappa+\beta / 2 \pi \kappa) \hat{e} \cdot\left(\frac{\vec{c}}{\ell}+\hat{e} \frac{\alpha}{2 \pi \omega \kappa}\right)} e^{\frac{i 2 \pi n}{p \ell}\left(q|c|+p N_{\Phi} c_{\gamma}\right)} e^{i \frac{\beta|c|}{\kappa \ell}} e^{i 2 \pi \frac{k_{1}|a|}{\ell N_{A}}}
$$


with the condition

$$
d_{\vec{c}+r \omega p^{2} \hat{e}}=d_{\vec{c}}
$$

The coefficients $D_{\vec{c}, n}$ satisfy the important property of being symmetric under a permutation of the entries of the vector $\vec{c}$.

We now give a resolution of the constraints on $\vec{c}$. The vector $\vec{c}$ satisfies the constraints if and only if there exist integers $m_{0}, m_{\gamma}$ with $0 \leq m_{0}<p$ such that the following equation is satisfied:

$$
c_{\gamma}=p N_{\Phi}\left(m_{0}+p m_{\gamma}\right)+\frac{q}{p \omega} \sum_{\delta=0}^{N_{A}}\left(c_{\gamma}-c_{\delta}\right)
$$

Given a solution $\vec{c}$ to the above equation, the eigenvalue $k_{2}$ can be expressed as:

$$
k_{2} \bmod N=\frac{1}{p^{2} \omega} \sum_{\delta=0}^{N_{A}}\left(c_{\delta}-c_{\gamma}\right)
$$

and $k_{1}$ can be picked to be any integer in the range $0 \leq k_{1}<N$ such that $k_{1} M=0 \bmod N$ where $M$ is defined to be the smallest integer in the range $0<M \leq N$ such that:

$$
\vec{c}+M r p^{2} \omega \hat{e}=\mathcal{P} \vec{c}+0 \bmod \ell Z^{N_{A}}
$$

for some $\mathcal{P} \in S_{N_{A}}$. That is, $M$ is the smallest integer such that the left hand side is a permutation of $\vec{c}$ (with the components of the vector defined $\bmod \ell$ ).

Let $A_{\gamma \delta}$ be the $N_{A} \times N_{A}$ matrix which acts on a vector by exchanging its $\gamma$ th and $\delta$ th entry. By a simple calculation one derives:

$$
\begin{aligned}
& \theta\left[\begin{array}{c}
\overrightarrow{0} \\
\vec{c} \\
\bar{\ell}
\end{array}\right]\left(A_{\gamma \delta} \vec{z}-\frac{\tau}{\omega \kappa}\left(n+\frac{\beta}{2 \pi}\right) \hat{e}+\frac{\alpha}{2 \pi \omega \kappa} \hat{e}, \Omega\right) \\
= & \theta\left[\begin{array}{c}
\overrightarrow{0} \\
\frac{A_{\gamma \delta} \vec{c}}{\ell}
\end{array}\right]\left(\vec{z}-\frac{\tau}{\omega \kappa}\left(n+\frac{\beta}{2 \pi}\right) \hat{e}+\frac{\alpha}{2 \pi \omega \kappa} \hat{e}, \Omega\right)
\end{aligned}
$$

Since any permutation can be written as a string of elementary transpositions $A_{\gamma \delta}$; given $\vec{c}$ satisfying equation (42) and ordered $\left(0 \leq c_{1} \leq \cdots \leq c_{N_{A}}<\ell\right)$, we can symmetrize the functions $F_{n, k_{1}, \vec{c}}$ :

$$
F_{n, k_{1}, \vec{c}}=\sum_{\mathcal{P} \in S_{N_{A}}} \sum_{K=0}^{M-1} D_{\vec{c}+K p^{2} r \omega, n} \theta\left[\begin{array}{c}
\overrightarrow{0} \\
\frac{\mathcal{P} \vec{c}}{\ell}+\frac{K}{N} \hat{e}
\end{array}\right]\left(\vec{z}-\frac{\tau}{\omega \kappa}\left(n+\frac{\beta}{2 \pi}\right) \hat{e}+\frac{\alpha}{2 \pi \omega \kappa} \hat{e}, \Omega\right)
$$

We are in a position to state the complete set of basis functions for $\psi$. Again assume $\vec{c}$ satisfies equation (42) and that its components are written in non-decreasing order, in addition assume that $0 \leq c_{1}<r p^{2} \omega$ to prevent counting basis elements more than once. A basis for the wave functions is then given by:

$$
\begin{aligned}
& \psi_{l, k_{1}, \vec{c}}=e^{-\frac{\pi \omega}{L_{1} L_{2}} \sum y_{\gamma}^{2}} e^{X v / L_{1}} \prod_{\gamma<\delta}\left|\theta_{1}\left(z_{\gamma}-z_{\delta} \mid \tau\right)\right|^{\frac{1}{\kappa}} \\
& \sum_{K=0}^{M-1} \sum_{n=0}^{p-1} \sum_{\mathcal{P} \in S_{N_{A}}} e^{i 2 \pi n\left(q|c|+p N_{\Phi} c_{\gamma}\right) / p \ell} e^{i 2 \pi K\left(n s / \kappa+\beta s / 2 \pi \kappa+k_{2} / N\right)} \\
& \theta\left[\begin{array}{c}
-\hat{e}(n / \kappa \beta / 2 \pi \kappa) \\
(K / N+\alpha / 2 \pi \omega \kappa) \hat{e}+\mathcal{P} \vec{c} / \ell
\end{array}\right](\vec{z}, \Omega) f_{l n}(v)
\end{aligned}
$$

\footnotetext{
${ }^{2}$ I thank Malin Ljungberg for her collaboration on this part of the paper
} 
where $k_{1}$ satisfies the condition stated above $\left(k_{1} M=0 \bmod N\right)$ and

$$
f_{l n}=\sum_{j=-\infty}^{\infty} e^{i \alpha(l+j q+n / \kappa)} \delta(\kappa v-2 \pi \kappa l-2 \pi(j p+n)-\beta)
$$

An irrelevant overall factor has been discarded in the above expression.

Next we turn to the special case of the exactly filled lowest Landau level, $N_{\Phi}=0$. In this particular case it is covenient to factorize the wave function as a product of a center of mass and relative wave function. P Write

$$
F_{n}(\vec{z})=h_{n}(Z) g\left(\vec{z}_{1}\right)
$$

where $h_{n}$ is holomorphic in $Z=(X+i Y) / L_{1}, g$ is independent of the index $n$ and depends only on the relative coordinates $\vec{z}_{1}, z_{1 \gamma}=z_{1}-z_{\gamma}$. The hamiltonian is periodic under translation along both non-trivial cycles when $N_{\Phi}=0$ and hence the magnetic translation operators become ordinary translation operators, and the wave functions become exactly periodic. We now prove that $g$ is a constant. To prove this result, fix $\gamma \neq 1$ and consider the center of mass preserving translations: $x_{\gamma} \rightarrow x_{\gamma}+L_{1}\left(N_{A}-1\right), x_{\delta} \rightarrow x_{\delta}-L_{1}(\delta \neq \gamma)$ and $y_{\gamma} \rightarrow y_{\gamma}+L_{2}\left(N_{A}-1\right)$, $y_{\delta} \rightarrow y_{\delta}-L_{2}(\delta \neq \gamma)$. Single valuedness of $\psi$ under both translations gives:

$$
\begin{gathered}
g\left(z_{1 \gamma}+N_{A},\left\{z_{1 \delta}\right\}_{\delta \neq \gamma}\right)=g\left(z_{1 \gamma},\left\{z_{1 \delta}\right\}_{\delta \neq \gamma}\right) \\
g\left(z_{1 \gamma}+N_{A} \tau,\left\{z_{1 \delta}\right\}_{\delta \neq \gamma}\right)=g\left(z_{1 \gamma},\left\{z_{1 \delta}\right\}_{\delta \neq \gamma}\right)
\end{gathered}
$$

That is, $g$ is a doubly periodic entire function in each of the $z_{1 \gamma}$. By Liouville's first theorem $g$ is a constant [8]. Periodicity of $\psi$ imposes constraints on the $h_{n}$

$$
\begin{gathered}
h_{n}(Z+1)=h_{n}(Z) \\
h_{n}(Z+\tau)=e^{-i \pi \frac{\tau}{\kappa}} e^{i \frac{\tau}{\kappa}(2 \pi n+\beta)} e^{-i \frac{\alpha}{\kappa}} h_{n-1}(Z) n>0 \\
h_{0}(Z+\tau)=e^{-i \pi \frac{\tau}{\kappa}} e^{i \frac{\tau}{\kappa} \beta} e^{-i \frac{\alpha}{\kappa}} e^{-i 2 \pi q Z} h_{p-1}(Z)
\end{gathered}
$$

which imply:

$$
h_{n}(Z+p \tau)=e^{-i \pi \tau p q} e^{i q \tau(2 \pi n+\beta)} e^{-i q \alpha} e^{-i 2 \pi q Z} h_{n}(Z)
$$

By an identical argument as the one presented for the $N_{\Phi} \neq 0$ case, the reader will easily convince herself that a basis for $h_{n}$ is provided by:

$$
\begin{aligned}
h_{n, c}= & e^{i \pi \tau \kappa\left(\frac{n}{\kappa}+\frac{\beta}{2 \pi \kappa}\right)^{2}} e^{-i 2 \pi\left(\frac{n}{\kappa}+\frac{\beta}{2 \pi \kappa}\right)\left(\frac{\alpha}{2 \pi}+\frac{c}{q}\right)} e^{-i 2 \pi c n / p} \\
& \theta\left[\begin{array}{c}
0 \\
\frac{c}{q}+\frac{\alpha}{2 \pi}
\end{array}\right](Z-\tau(n+\beta / 2 \pi)+\alpha / 2 \pi, \tau \kappa)
\end{aligned}
$$

where $c$ is an integer in the range $0 \leq c<q$. The $q$-fold degenerate set of wave functions can be written as:

$$
\begin{aligned}
\psi_{l, c}= & e^{-\pi \frac{\omega}{L_{1} L_{2}} \sum y_{\gamma}^{2}} e^{X v / L_{1}} \prod_{\gamma<\delta}\left|\theta_{1}\left(z_{\gamma}-z_{\delta} \mid \tau\right)\right|^{\frac{1}{\kappa}} e^{i X v} \\
& \sum_{n=0}^{p-1} e^{i 2 \pi c n / p} \theta\left[\begin{array}{c}
-(n / \kappa+\beta / 2 \pi \kappa) \\
c / q+\alpha / 2 \pi
\end{array}\right](Z, \tau \kappa) f_{l n}(v)
\end{aligned}
$$

\footnotetext{
${ }^{3}$ Note that this is not always possible. It is only possible if $N_{\Phi}=0$ or in the case $N_{\Phi} \neq 0$ when $s=1$, i.e. when $N_{\Phi}$ is divisible by $N_{A}$.
} 
where $f_{l n}$ was defined above. An irrelevant constant factor has once again been discarded.

Finally, we perform a gauge transformation to bring the hamiltonian to a form which describes anyons in an external magnetic field with no long-range Aharonov-Bohm interactions. Define the wave function $\tilde{\psi}_{l}^{0}[1]$ as:

$$
\psi_{l}=\prod_{\gamma<\delta}\left(\frac{\theta_{1}^{*}\left(z_{\gamma}-z_{\delta} \mid \tau\right)}{\theta_{1}\left(z_{\gamma}-z_{\delta} \mid \tau\right)}\right)^{\frac{1}{2 \kappa}} e^{i X v} \tilde{\psi}_{l}^{0}
$$

This gauge transformation removes the long range interaction between particles keeping only the interaction with the external magnetic field. $\tilde{\psi}_{l}^{0}$ picks up the correct phase when two particle coordinates are exchanged. Let us investigate how the wave function transforms under translations by a lattice vector. It will be convenient to define the following $p$ set of wave functions, for $N_{\Phi} \neq 0$ :

$$
\begin{aligned}
& \tilde{\psi}_{l, k_{1}, \vec{c}}^{j}=e^{-\frac{\pi \omega}{L_{1} L_{2}} \sum y_{\gamma}^{2}} \prod_{\gamma<\delta}\left(\theta_{1}\left(z_{\gamma}-z_{\delta} \mid \tau\right)\right)^{\frac{1}{\kappa}} \\
& \sum_{K=0}^{M-1} \sum_{n=0}^{p-1} \sum_{\mathcal{P} \in S_{N_{A}}} e^{-i 2 \pi \frac{n j}{\kappa}} e^{i 2 \pi n\left(q|c|+p N_{\Phi} c_{\gamma}\right) / p \ell} e^{i 2 \pi K\left(n s / \kappa+\beta s / 2 \pi \kappa+k_{2} / N\right)} \\
& \theta\left[\begin{array}{c}
-\hat{e}(n / \kappa \beta / 2 \pi \kappa) \\
(K / N+\alpha / 2 \pi \omega \kappa) \hat{e}+\mathcal{P} \vec{c} / \ell
\end{array}\right](\vec{z}, \Omega) f_{l n}(v)
\end{aligned}
$$

and for $N_{\Phi}=0$

$$
\begin{aligned}
\psi_{l, c}= & e^{-\pi \frac{\omega}{L_{1} L_{2}} \sum y_{\gamma}^{2}} \prod_{\gamma<\delta}\left(\theta_{1}\left(z_{\gamma}-z_{\delta} \mid \tau\right)\right)^{\frac{1}{\kappa}} e^{i X v} \\
& \sum_{n=0}^{p-1} e^{i 2 \pi c n / p} e^{-i 2 \pi \frac{j n}{\kappa}} \theta\left[\begin{array}{c}
-(n / \kappa+\beta / 2 \pi \kappa) \\
c / q+\alpha / 2 \pi
\end{array}\right](Z, \tau \kappa) f_{l n}(v)
\end{aligned}
$$

Now under a translation $x_{\alpha} \rightarrow x_{\alpha}+L_{1}$, while holding the other coordinates fixed, the wave function changes its component index as follows:

$$
\tilde{\psi}_{l}^{j}\left(x_{\alpha}+L_{1}\right)=e^{-i \frac{\beta}{\kappa}+i \frac{\pi}{\kappa}\left(N_{A}-1\right)} \tilde{\psi}_{l}^{j+1}\left(x_{\alpha}\right)
$$

A translation $y_{\alpha} \rightarrow y_{\alpha}+L_{2}$ does not affect the component index of the wave function but it picks up a component dependent phase:

$$
\tilde{\psi}_{l}^{j}\left(y_{\alpha}+L_{1}\right)=e^{i 2 \pi \omega x_{\alpha} / L_{1}} e^{i 2 \pi \frac{j}{\kappa}-i \frac{\pi}{\kappa}\left(N_{A}-1\right)} \tilde{\psi}_{l}^{j+1}\left(y_{\alpha}\right)
$$

Where the degeneracy indices have been suppressed. There are three important points to note here: 1) the wave function picks up the correct gauge transformation under translation since now only the external magnetic field is present, the C-S contribution has been "gauged" away; 2) the wave function has $p$ components; 3 ) the components survive even when $\omega$ is itself an integer (that is, the components are not an artifact of the non-commutativity of the appropriate magnetic translation operators). Finally, it is interesting to observe that when $N_{\Phi}=0$ the ground state is non-degenerate only when $q=1$. So statistics of the type $\pi / p$ are special in that they have non-degenerate ground states when the filling factor is exactly one. Moreover, the $N_{\Phi}=0$ states are of the Laughlin-Jastrow form investigated by Haldane and Rezayi in the fermion case [13. 
To summarize, we have found the complete set of ground state wave functions for $N_{A} / \omega \kappa \leq 1$. The wave functions have $p$ components consistent with both arguments based on the braid group and the statistics transmuting Chern-Simons field [10]. Though we have provided a complete basis of wave functions we have been unable to give a closed expression for the total degeneracy for the case $N_{\Phi} \neq 0$. This is due to the rather complicated constraints satisfied by the vector $\vec{c}$. A more transparent resolution of the constraint may be possible allowing one to give a closed expression for the degeneracy.

I thank T. H. Hansson, A. Karlhede, M. Ljungberg and E. Westerberg for illuminating discussions.

\section{References}

[1] J. M. Leinaas and J. Myrheim, Nuovo Cimento B37, 1 (1977); F. Wilczek, Phys. Rev. Lett. 49, 957 (1982).

[2] R. B. Laughlin, Phys. Rev. Lett. 50, 1395 (1983); F. D. M. Haldane, Phys. Rev. Lett. 51, 605 (1983).

[3] B. I. Halperin, Phys. Rev. Lett. 52, 1583 (1983); D. Arovas, J. R. Shrieffer and F. Wilczek, Phys. Rev. Lett. 53, 772 (1984).

[4] See for instance: G. V. Dunne, A. Lerda, S. Sciuto and C. A. Trugenberger Nucl. Phys. B370, 601 (1990); A. Karlhede and E. Westerberg, Int. J. Mod. Phys. B6, 1595 (1992); A. P. Polychronakos, Phys. Lett. B264, 362 (1990).

[5] S. Randjbar-Daemi, A. Salam, J. Strathdee, Phys. Lett. B240, 121 (1990).

[6] R. Iengo and K. Lechner, Nucl. Phys. B346, 551 (1990); K. Lechner, Anyon Physics on the Torus, Thesis, (1991).

[7] D. Mumford, Tata Lectures on Theta, vol. I, Birkhäuser, Boston, 1983.

[8] K. Knopp, Theory of Functions, Part II, Dover, New York, 1974.

[9] F. D. M. Haldane, Phys. Rev. Lett. 55, 2095 (1985).

[10] T. Einarsson, Phys. Rev. Lett. 64, 1995 (1990); X. G. Wen et. al., Phys. Rev. B42, 6110 (1990); T. Imbo and J. March-Russell, Phys. Lett. B252, 84 (1990) ; K. Lee, "Anyons on Spheres and Tori", BU/HEP-89-28.

[11] A. Fayyazuddin, "On the origin of multi-component anyon wave functions", Stockholm preprint, USITP-92-15 (to appear in Nuc. Phys.B).

[12] C-L Ho and Y. Hosotani, Int. Jour. Mod. Phys. A7, 5797 (1992).

[13] F. D. M. Haldane and Rezayi, Phys. Rev. B31, 2529 (1985). 